IAU Colloquium 164: Radio Emission from Galactic and Extragalactic Compact Sources

ASP Conference Series, Vol. 144, 1998

J. A. Zensus, G. B. Taylor, \&J. M. Wrobel (eds.)

\title{
Relativistic Jets in Low Power Radio Galaxies
}

G. Giovannini ${ }^{1}$, E. Arbizzani, L. Feretti, \& T. Venturi

Istituto di Radioastronomia, 40129 Bologna, Italy

W. D. Cotton

National Radio Astronomy Observatory, Charlottesville, VA 22903, U.S.A.

L. Lara

Instituto de Astrofisica de Andalucia, CSIC, 18080 Granada, Spain

G. B. Taylor

National Radio Astronomy Observatory, Socorro, NM 87801, U.S.A.

Abstract. From VLBI observations of $11 \mathrm{FR} \mathrm{I} \mathrm{radio} \mathrm{galaxies} \mathrm{we} \mathrm{find} \mathrm{that:} \mathrm{1.} \mathrm{parsec} \mathrm{scale}$ jets are relativistic; 2 . 3C 264 shows a relativistic jet decelerating moving from the core to the extended lobes; 3. $3 \mathbf{C ~} 338$ is a source with a symmetric parsec scale structure and morphological changes, implying proper motion on both sides of the source; $4.1144+35$ is an extended low power radio galaxy with an apparent superluminal motion in its parsec scale radio structure.

\section{Evidence of Relativistic Jets}

We have observed with VLBI, 11 extended low power radio galaxies (FR I) from a complete sample under study by us (see Giovannini et al. 1990 for the sample definition and Lara et al. 1997a for recent results and references).

From observational data, we have derived constraints on the jet velocity and orientation with respect to the line of sight (see Giovannini et al. 1994). In Table 1 observed radio galaxies are presented in decreasing order of their total radio power at $408 \mathrm{MHz}$ (we use $\mathrm{H}_{0}=100 \mathrm{~km} \mathrm{sec}^{-1} \mathrm{Mpc}^{-1}$ ). The lower limits of the Lorentz factor $\gamma$ show that parsec scale jets are relativistic in agreement with the unified scheme for low power sources.

3C 264: This galaxy shows an optical jet due to synchrotron emission (Lara et al. 1997b). We modeled the jet brightness as the combined effects of Doppler boosting and adiabatic losses, as traced through the jet velocity and width (Baum et al. 1997). The best fit was obtained with a fast jet $(\mathrm{v} \sim 0.98 \mathrm{c})$ in the inner $250 \mathrm{pc}$ from the core. At this distance we have evidence of interaction between the jet and the surrounding medium, producing in the jet a rapid deceleration to $\sim 0.4 \mathrm{c}$. At $\sim 300 \mathrm{pc}$ from the core, the jet begins a slow but steady deceleration.

3C 338 This source shows at parsec and kpc scale a two-sided symmetric structure which is consistent with a source orientation very close to the plane of the sky (Feretti et al. 1993; Giovannini et al. in preparation). Multi-epoch observations show evident morphological changes, implying a source proper motion with apparent velocity $\sim 0.4 \mathrm{c}$. However, the measured proper motion is crucially dependent on the Hubble constant. If $\mathrm{H}_{0}$ is $50 \mathrm{~km} \mathrm{~s}^{-1} \mathrm{Mpc}^{-1}$, the jet velocity is $\sim 0.8 \mathrm{c}$ and the Doppler factor is $\sim 0.64$. If this is the case, the intrinsic flux density of the parsec scale structure would be higher than observed due to the

\footnotetext{
${ }^{1}$ Dipartimento di Astronomia, Universitá di Bologna, 40126 Bologna, Italy
} 
Table 1. Observed Radio Galaxies.

\begin{tabular}{lcccc}
\hline \multicolumn{3}{c}{ Table 1. Observed Radio Galaxies. } & \\
\hline IAU Name & Name & $\begin{array}{c}(1) \\
\mathrm{z}\end{array}$ & $\begin{array}{c}(2) \\
\text { Log } P_{\text {tot }} \\
\text { W/Hz }\end{array}$ & $\begin{array}{c}(3) \\
\gamma_{\text {min }}\end{array}$ \\
\hline $2335+26$ & 3C 465 & 0.0301 & 25.30 & 1.35 \\
$1626+39$ & 3C 338 & 0.0303 & 25.25 & 1.09 \\
$0836+29$ & & 0.0790 & 25.08 & 2.29 \\
$0755+37$ & NGC 2484 & 0.0413 & 25.04 & 1.40 \\
$0220+43$ & 3C 66B & 0.0215 & 24.98 & 1.63 \\
$0206+35$ & 4C 35.03 & 0.0375 & 24.85 & 1.21 \\
$1142+20$ & 3C 264 & 0.0206 & 24.85 & 1.32 \\
$0104+32$ & 3C 31 & 0.0169 & 24.50 & 1.27 \\
$1144+35$ & & 0.0630 & 24.03 & 1.72 \\
$0055+30$ & NGC 315 & 0.0167 & 23.95 & 1.60 \\
$1322+36$ & NGC 5141 & 0.0175 & 23.75 & 1.28 \\
\hline
\end{tabular}

Notes: 1: Redshift; 2: Log. of the total radio power at $408 \mathrm{MHz} ; 3:$ Min. Lorentz factor.

de-boosting by the relativistic motion, but still in agreement with values found in other FR I sources.

$1144+35$ This source shows an extension of more than $200 \mathrm{kpc}$ in the NVSS survey data. At parsec resolution, 5 observations spread over five years at 5 $\mathrm{GHz}$ show a faint nuclear emission with two short symmetric jets, and two blobs moving at an apparent velocity of $1.7 \mathrm{c}$ from the core (Giovannini et al. in preparation). This is the first low power source after M87 where an apparent superluminal motion is firmly established. The parsec scale structure, the superluminal motion, and the large extent of the whole source suggest the presence of a complex geometry.

Acknowledgments. The National Radio Astronomy Observatory is a facility of the National Science Foundation, operated under a cooperative agreement by Associated Universities, Inc.

\section{References}

Baum, S.A., et al. 1997. $A p J, 483$ 178-193.

Feretti, L., et al. 1993. $A p J, 408,446-451$.

Giovannini, G., et al. 1990. $A p J, 358,159-163$.

Giovannini, G., et al. 1994. $A p J, 435,116-127$.

Lara, L., et al. 1997a. $A p J, 474,179-187$.

Lara, L., et al. 1997b. Vistas in Astronomy, 41, 241-245. 